\title{
How Anomalous Resistivity Accelerates Magnetic Reconnection
}

H. Che

University of Maryland, College Park, MD, 20742, USA and

NASA Goddard Space Flight Center, Greenbelt, MD, 207r1, $U S A$

(Dated: 11 August 2017)

Whether turbulence induced anomalous resistivity (AR) can facilitate a fast magnetic reconnection in collisionless plasma is a subject of active debate for decades. Recent space observations suggest that the reconnection rate can be higher than the Hall-reconnection rate and turbulent dissipation is required. In this paper, using particle-in-cell simulations, we present a case study of how AR produced by Buneman instability accelerates magnetic reconnection. We first show that the AR/drag produced by Buneman instability in a thin electron current layer 1) can dissipate magnetic energy stored in the current layer through dissipation of the kinetic energy of electron beams; 2) The inhomogeneous drag caused by wave couplings spontaneously breaks the magnetic field lines and causes impulsive fast non-Hall magnetic reconnection on electron-scales with a mean rate reaching $0.6 V_{A}$. We then show that a Buneman instability driven by intense electron beams around the $\mathrm{x}$-point in a 3D magnetic reconnection significantly enhances the dissipation of the magnetic energy. Electron-scale magnetic reconnections driven by the inhomogeneous drag around the $\mathrm{x}$-line enhances the reconnection electric field and the in-plane perpendicular magnetic field. About $40 \%$ of the released magnetic energy is converted into the electron thermal energy by AR while $50 \%$ is converted into the kinetic energy of the electron beams through the acceleration by the reconnection electric field. The enhanced magnetic energy dissipation is balanced by a net Poynting flux in-flow. About $10 \%$ of the released magnetic energy is brought out by an enhanced Poynting flux out-flow. These results suggest that AR with sufficient intensity and electron-scale inhomogeneity can significantly accelerate magnetic reconnection. 


\section{INTRODUCTION}

In the Earth's magnetosphere ${ }^{1-5}$ and solar and stellar atmospheres ${ }^{6-8}$, plasma heating commonly occurs in current sheets of various scales. Different types of macro and microinstabilities, including both electrostatic(ES) and electromagnetic(EM) instabilities, can be triggered in the current sheets. Some can lead to the merging and rearranging of oppositely directed magnetic field lines and bursty releasing of magnetic energy - a process known as magnetic reconnection.

In magnetic reconnections, the breaking of magnetic field lines requires the ideal magnetohydrodynamics (MHD) frozen-in condition $\mathbf{E}+\mathbf{U} \times \mathbf{B}=0$ to be broken. In resistive MHD theory, e.g. the established Sweet-Parker model ${ }^{9}$, it is the collisional resistivity $\eta$ that breaks the frozen-in condition, and the Ohm's law assumes the well known form $\mathbf{E}+\mathbf{U} \times \mathbf{B}=\eta \mathbf{j}$. However, the collisional resistivity of space plasmas is usually too low to facilitate a sufficiently fast Sweet-Parker magnetic reconnection to explain the observed magnetic energy release in solar flares and magnetospheric substorms.

In thin current sheets, ions and electrons decouple and two-fluid effects dominate ${ }^{10,11}$. In this case the Ohm's law is replaced by the generalized Ohm's law - the first moment of electron Vlasov equation ${ }^{12,13}$ :

$$
\mathbf{E}+\frac{1}{c} \mathbf{U}_{i} \times \mathbf{B}=-\frac{m_{e}}{e}\left(\partial_{t} \mathbf{U}_{e}+\mathbf{U}_{e} \nabla \cdot \mathbf{U}_{e}\right)+\frac{1}{e n_{e} c} \mathbf{j} \times \mathbf{B}-\frac{1}{e n_{e}} \nabla \cdot \mathbb{P}_{e}-\eta \mathbf{j}_{e},
$$

where we used $\mathbf{U}_{e} \times \mathbf{B} / c \equiv\left(\mathbf{U}_{i}-\mathbf{j} /\right.$ en $\left._{e}\right) \times \mathbf{B} / c$ and for simplicity assumed the plasma being fully ionized hydrogen with $n_{e}=n_{i}$ where $n_{i}$ and $n_{e}$ are ion the electron density respectively. The Hall term ${ }^{14}$ is defined as $\mathbf{j} \times \mathbf{B} / c$. Besides the electron Joule heating $\eta \mathbf{j}_{e}$, the generalized Ohm's law presents more terms that can break the frozen-in condition, including the gradient of electron pressure $\mathbb{P}_{e}$ and the electron inertia terms (acceleration $\partial_{t} \mathbf{U}_{e}$ and convective momentum $\mathbf{U}_{e} \nabla \cdot \mathbf{U}_{e}$ ). The Hall term increases as the spatial scale approaches the ion inertial length $d_{i}=c / \omega_{p i}$ (where $\omega_{p i}=\sqrt{m_{i} / 4 \pi e^{2} n_{e}}$ ) but decreases as the spatial scale decreases to the electron inertial length $d_{e}=c / \omega_{p e}$. The Hall term becomes zero at the x-point and thus it can not break magnetic field lines but rather helps to form an $\mathrm{x}$-point configuration through the Hall field ${ }^{10,15,16}$. The inertia and pressure terms are important on electron scales ${ }^{12,17-19}$.

Simulations of laminar driven magnetic reconnection, e.g. the GEM magnetic reconnection challenge ${ }^{12}$, show that by including Hall-effect reconnection can become dramatically 
faster than Sweet-Parker reconnection, and its maximum steady reconnection rate can reach 0.1-0.2 $V_{A}$, where $V_{A}$ is Alfvén speed. An interesting observation based on a set of particlein-cell (PIC) simulations is that the maximum Hall-reconnection rate seems universal and is independent of the mechanism that breaks the field lines ${ }^{20,21}$. Observations and laboratory experiments have discovered the Hall field and Hall current associated with fast collisionless magnetic reconnection ${ }^{10,12}$, but no direct experimental or observational evidence has been found to show that the rate is irrelevant to the dissipation mechanism ${ }^{22-25}$. On the other hand, observations of solar flares found that magnetic reconnection may commonly be turbulent as evidenced by the filamentary structure of magnetic field, non-thermal heating observed in X-ray, and coherent radio emissions that are likely results of electron two-stream instability $^{26-28}$. The reconnection rate in solar flares estimated by the variability of magnetic flux around magnetic null-points varies in a wide range, from the moderate $0.1 V_{A}$ to very fast $0.5-0.6 V_{A}^{29}$ - much higher than the maximum Hall-reconnection rate from simulations. Note that solar flare magnetic reconnections mostly have strong guide magnetic field. Experiments ${ }^{30}$ and simulations ${ }^{31}$ show that the reconnection rate of guide field reconnection is lower than the anti-parallel reconnection rate. Therefore the discrepancy between the simulation results and observation is more severe than it appears. New in situ Magnetospheric Multiscale Science (MMS) observations of magnetopause reconnection provide the first direct evidence that the reconnection rate is higher than the Hall-reconnection rate and the turbulence induced anomalous resistivity is probably required to explain the enhanced reconnection rate $^{32}$. In addition, Magnetic Reconnection Experiment (MRX) suggests that anomalous resistivity may be able to facilitate a fast Sweet-Parker-like magnetic reconnection ${ }^{33,34}$.

It's not surprising that the inconsistency exists between observations and numerical simulations. First, past numerical simulations have focused on a class of driven magnetic reconnections with a limited parameter space ${ }^{12}$ which may not be representative of the reconnections in nature; Second, the simulated reconnections can be affected by initializations and boundary condition choices and it is unclear how these affect magnetic reconnection rate and the generation of instabilities and waves on different scales ${ }^{35}$. A recent simulation carried out by Sitnov et al. shows that an internal instability drives a new reconnection in the ion diffusion region (IDR) with an unreported Hall-field pattern ${ }^{36}$, while a simulation carried out by Che et al. found that kinetic instabilities driven at the reconnection $\mathrm{x}$-line can 
significantly increase the reconnection rate $^{37}$. In nature, the ways magnetic reconnection can be triggered are far more diverse than have been explored in simulations, and the process can occur on a wide range of scales simultaneously. More importantly, observations of both magnetospheric magnetic reconnections and solar flares show that magnetic reconnection are generally impulsive and turbulent ${ }^{22,27,38,39}$. While the theory of collisional magnetic reconnection is better established, turbulent magnetic reconnection which involves wave-wave and wave-particle interactions on a broad range of spatial and time scales is still poorly understood.

What is known is that the turbulence effects such as anomalous resistivity (AR) and anomalous viscosity (AV) can dramatically influence the energy dissipation and convective momentum transport. Thus the generalized Ohm's law in Eq. (1) needs to be modified for turbulent reconnection ${ }^{37}$ :

$$
\langle\mathbf{E}\rangle+\left\langle\mathbf{U}_{e}\right\rangle \times\langle\mathbf{B}\rangle / c=\mathbf{D}_{e}+\nabla_{\perp} \cdot \boldsymbol{\Xi}_{e}-\frac{m_{e}}{e}\left(\partial_{t}\left\langle\mathbf{U}_{e}\right\rangle+\left\langle\mathbf{U}_{e} \partial \cdot \mathbf{U}_{e}\right\rangle\right)-\frac{1}{e\left\langle n_{e}\right\rangle} \partial \cdot\left\langle\mathbb{P}_{e}\right\rangle .
$$

where $\mathbf{D}_{e} \equiv-\left\langle\delta n_{e} \delta \mathbf{E}\right\rangle /\left\langle n_{e}\right\rangle$ is the drag, i.e. AR, $\boldsymbol{\Xi}_{e} \equiv-\left\langle\delta \mathbf{p}_{e}\left(\delta \mathbf{U}_{e}-e \delta \mathbf{A} / m_{e} c\right)\right\rangle / e\left\langle n_{e}\right\rangle$ is $\mathrm{AV}, \mathbf{p}_{e}=n_{e} \mathbf{U}_{e}$, and $\mathbf{A}$ is the magnetic vector potential. $\langle\ldots\rangle$ denotes the ensemble average of the turbulence properties.

$\mathrm{AR}^{40}$ is induced by ES instabilities, arising from ion-electron drag, and $A V^{41}$ is induced by EM instabilities, arising from anomalous momentum transport. Whether AR and AV can facilitate fast magnetic reconnection in collisionless plasma (if so, how it is achieved) is a fundamental problem in both plasma physics and space physics and is a subject of debate for nearly half a century ${ }^{34,37,42-53}$. The problem is particularly difficult because AR and AV are highly nonlinear processes which take part in the even more complex magnetic reconnection processes. Diagnosing the role of $\mathrm{AR}$ and $\mathrm{AV}$ in magnetic reconnection is a daunting task, and the progress is slow. In observations, since kinetic instabilities generating $\mathrm{AR}$ and $\mathrm{AV}$ are dominated by electron dynamics, addressing the problem experimentally requires resolution of the electron diffusion region (EDR). Only recently it becomes possible to probe such scales with the launch of the MMS.

The analysis of the generalized Ohm's Law (Eq. 1) using the observational data from the first encounter of a magnetopause reconnection EDR by MMS shows that AR is required to explain the enhanced magnetic reconnection rate $^{32}$. On the other hand, many other MMS observations discovered coherent electrostatic structures and electron heating in reconnection 
diffusion region, implying that ES instabilities are common in reconnections ${ }^{32,54-58}$. Thus it is necessary for us to revisit how AR affects the process of magnetic reconnection in the EDR, and how AR couples with Hall effect to affect the IDR - both are fundamental problems in plasma physics ${ }^{59}$.

In this paper, we concentrate on $\mathrm{AR}$ induced by Buneman instability in an unsteady guide-field reconnection. Buneman instability is triggered due to the intense electron beams developed at the x-line in magnetic reconnection and once the electron beam velocity is larger than the electron thermal velocity ${ }^{40,45,60-63}$. Recent MMS observations of magnetospheric reconnection discovered that Buneman instability can efficiently brake the electron jet at the exhaust of the diffusion region ${ }^{54}$. Electron holes driven by Buneman instability are often discovered to associate with electron-scale current sheets in reconnections in the magnetosphere ${ }^{54,56,64-68}$. Intense thin electron current sheet develops near the $\mathrm{x}$-line in the EDR has also been discovered for the first time by $\mathrm{MMS}^{69}$.

The 3D guide field magnetic reconnection simulation first published by Che et al. $(2011)^{37}$ is so far the only study that clearly demonstrates the dramatic enhancement of reconnection rate by turbulence, because in this particular case turbulence effects can be separated from Hall-effect by comparing 3D PIC simulations with a 2D benchmark simulation. In this 3D guide field magnetic reconnection simulation, intense electron beam develops at late stage when the current sheet near the x-line becomes very thin (with width $\sim d_{e}$ ) and triggers a Buneman instability. At the late stage of the reconnection, the nonlinear evolution of Buneman instability triggers whistler wave turbulence and then induces anomalous viscosity that maintain the fast reconnection ${ }^{37}$. Careful analysis of this simulation can enable us to gain better insights into how turbulence effects accelerate magnetic reconnections.

The outline of the paper is as follows: In $\S$ II A we first describe the simulations used in this analysis; then in $\S$ II B we present an analysis of Buneman instability induced AR in a thin current layer, in which we show the inhomogeneous drag can spontaneously break magnetic field lines to produce electron scale magnetic reconnections that are not affected by the Hall-effect. In $\S$ II C we examine the properties of the Buneman instability induced drag in a 3D force-free magnetic reconnection and show how drag helps to break the field lines, accelerate magnetic reconnection, and dissipate magnetic energy. The conclusions and a discussion are presented in $\S$ III. 


\section{SIMULATIONS AND ANALYSIS}

\section{A. Simulations}

Four PIC simulations using the P3D $\operatorname{code}^{70}$ are employed in this analysis, including simulations of Buneman instability in a thin current layer in 2D and 3D, and magnetic reconnection also in $2 \mathrm{D}$ and $3 \mathrm{D}$.

The initialization of these simulations are similar to or based on previous papers ${ }^{37,51,71}$. The differences between the Buneman and reconnection simulations are 1) the width of the initial current sheet in the former is of electron inertial length scale $d_{e}$ while the latter is of ion inertial length scale $d_{i}$; and 2) contrary to reconnection simulations, no initial perturbation is applied in the Buneman instability simulation so that the Buneman instability can develop well before magnetic reconnection starts spontaneously.

The parameters of 3D Buneman instability are the same as those in the simulation reported previously ${ }^{51}$. The initial magnetic field has a force-free configuration with $B_{x, 0} / B_{0}=$ $\tanh \left[\left(y-L_{y} / 2\right) / w_{0}\right]$, where $w_{0}$ and $L_{y}$ are the half-width of the initial current sheet and the simulation box size in $y$-direction, respectively. The guide magnetic field $B_{g}^{2}=B_{z, 0}^{2}=$ $B_{0}^{2}-B_{x, 0}^{2}$ is chosen so that the total magnetic field $|B| \equiv \sqrt{26} B_{0}$. We choose $w_{0}=d_{e}$ in the Buneman instability simulations and $w_{0}=0.5 d_{i}$ in the magnetic reconnection simulations. Simulations use periodic boundary conditions in the $x$ and $z$ directions, and conducting boundary condition in the $y$ direction. The initial plasma temperature and density are isotropic and uniform, with $T_{e 0}=T_{i 0}=0.04 m_{i} V_{A 0}^{2}$, where $V_{A 0}=B_{0} /\left(4 \pi n_{0} m_{i}\right)^{1 / 2}$ is the asymptotic ion Alfvén speed. A mass ratio $m_{i} / m_{e}=100$ is used in all simulations. The force-free condition requires $\mathbf{j}_{e} \times \mathbf{B}=0$, thus initially $j_{e x} / j_{e z}=B_{x} / B_{z}$.

The domain of 3D simulation of Buneman instability has dimensions $L_{x} \times L_{y} \times L_{z}=1 d_{i} \times$ $1 d_{i} \times 2 d_{i}$ with grid number $512 \times 512 \times 1024$. The particle number per cell is 100 . The initial electron drift velocity is $v_{d e} \sim 9 V_{A 0} \sim 3 v_{t e z, 0}$, which is large enough to trigger electrostatic Buneman instability. The total simulation time is $\omega_{p e, 0} t=160$ where $\omega_{p e, 0} \equiv\left(4 \pi n_{0} e^{2} / m_{e}\right)^{1 / 2}$ is the initial electron plasma frequency. The same initialization is used in the corresponding $2 \mathrm{D}$ simulation except that only 1 cell is used in the $\mathrm{x}$-direction. The $2 \mathrm{D}$ simulation is in the $\mathrm{y}-\mathrm{z}$ plane in which the waves of Buneman instability propagate along $\mathrm{z}$ and form $2 \mathrm{D}$ electron holes in the $\mathrm{y}-\mathrm{z}$ plane. 
The parameters for the 3D magnetic reconnection simulation are the same as those in the simulation published in a previous paper ${ }^{37}$. The domain has dimensions $L_{x} \times L_{y} \times L_{z}=$ $4 d_{i} \times 2 d_{i} \times 8 d_{i}$ with grid number $512 \times 256 \times 1024$. The particle number per cell is 20 . The initial temperature is the same as that in the Buneman instability simulation, but the initial electron drift is $v_{d e} \sim 4 V_{A 0} \sim v_{t e, 0}$, much smaller than that in the Buneman instability simulation, thus the Buneman instability is much weaker in the $3 \mathrm{D}$ reconnection simulation. The total simulation time is $\Omega_{i 0} t=4$, where $\Omega_{i 0}$ is the asymptotic ion gyro-frequency and $\omega_{p e, 0} / \Omega_{i 0}=200$. The corresponding $2 \mathrm{D}$ reconnection domain is $L_{x} \times L_{y}=4 d_{i} \times 2 d_{i}$. The total simulation time $\Omega_{i 0} t=4$ is short enough to prevent the inflow $V_{i n}$ and outflow recirculation due to $V_{\text {in }}<<V_{\text {out }}<V_{A 0}$ and the passing time of the flow is $<<4 d_{i} / V_{A 0}=4 \Omega_{i 0}^{-1}$.

The simulation quantities are dimensionless, with magnetic field normalized to the asymptotic $B_{0}$, density to the asymptotic $n_{0}$, and velocity to $V_{A 0}$. The units of time and distance are $\Omega_{i 0}^{-1}$ (or $\omega_{p e, 0}^{-1}$ in some cases) and $d_{i}$ respectively. In the dimensionless units, electric field $E_{0}=V_{A 0} B_{0} / c$.

\section{B. Buneman Instability in a thin current sheet and the Spontaneous Fast Electron-Scale Magnetic Reconnection}

In the $3 \mathrm{D}$ simulation of the thin current sheet a Buneman instability is driven along the magnetic field (which is close to the $z$-direction) at $\omega_{p e, 0} t \sim 40$ with the initial growth rate $\sqrt{3} / 2\left(m_{e} / 2 m_{i}\right)^{1 / 3} \omega_{p e, 0} \sim 0.1 \omega_{p e, 0}$, consistent with the linear growth rate of Buneman instability in cold plasma. The electric fluctuation $\left\langle\delta E_{z}^{2}\right\rangle^{1 / 2}(\langle\ldots\rangle$ denotes the average over z, which approximates the ensemble average) generated by the Buneman instability reaches its peak at $\omega_{p e, 0} t \sim 70$ and then decays and saturates at $\omega_{p e, 0} t \sim 160$. As the magnitude of electric fluctuations become large enough to trap electrons and form electron holes with electric potential satisfying $\phi>k T_{e}$, the Buneman instability enters the nonlinear stage and the growth rate decreases due to thermal effects ${ }^{71}$. The fast adiabatic energy exchange between the electron holes and the trapped electrons causes rapid phase mixing and heating, leading to the decay of waves and the de-trapping of electrons until the saturation of the Buneman turbulence when the electron holes break up ${ }^{71}$. Previous study ${ }^{51}$ shows that the kinetic energy of the current and the associated magnetic energy are dissipated by AR in the form of drag $\mathbf{D}_{e}$. The dissipated kinetic energy is converted into parallel electron heating 
while the dissipated magnetic energy is converted into perpendicular electron heating. At the same time, besides the electron holes, an inductive electric field is produced due to the dissipation of magnetic energy.

A natural question is whether AR can break magnetic field lines as the magnetic energy is dissipated. Indeed, after the Buneman instability is triggered at $\omega_{p e, 0} t \sim 40$, electron-scale magnetic reconnections appear at different locations along the mid-plane of the current sheet in the simulation. Examples of the magnetic field lines averaged over $\mathrm{z}$ at $\omega_{p e, 0} t=48,60$ are shown in Fig. 1. Magnetic field perpendicular to the current sheet $\left\langle B_{y}\right\rangle$, which was originally zero, is generated at $x=0.7 d_{i}$ and an x-line is formed at $\omega_{p e, 0} t=60$. A clear manifestation of topological change of the magnetic fields is shown in the magnetic vector potential $\left\langle A_{z}\right\rangle$ with amplitude varying from 0 to $-0.5 B_{0} / d_{i}$ (Fig.2, multimedia view). As a result of the magnetic reconnection, the inductive electric field $\left\langle E_{z}\right\rangle$ is produced with a value as high as $0.6 V_{A 0} B_{0} / c$. The corresponding localized intense electric field $E_{z}$ increases to as high as $40 E_{0}$ and the electron density fluctuations can be as high as $\delta n / n_{0} \sim 0.8$ in the mid-plane, creating the observed intense $\operatorname{drag}\left\langle\delta n_{e} \delta E_{z}\right\rangle$.

A remarkable feature of the electron-scale magnetic reconnection is that contrary to ionscale reconnection, contribution from Hall-effect is negligible. In Hall-reconnections the Hall effect generates an out-of-plane Hall magnetic field ${ }^{10,15}$. Hall field has a quadrupole structure in anti-parallel reconnections ${ }^{16}$, and the quadrupole is distorted in guide-field reconnections ${ }^{31,72}$. Such a quadrupole Hall field is clearly absent in the simulation as shown in the out-of-plane magnetic field $\left\langle B_{z}\right\rangle-B_{g}$ produced during the Buneman instability (Fig. 1). What we observe is a very small decrease of $B_{z}$ caused by the dissipation of $j_{e x}\left(<<j_{e z}\right)$. Hall effect is produced due to the decoupling of ions and electrons. The reason for the disappearance of Hall effect is that the electrons are fully demagnetized inside the electron holes and the wave-electron interactions on electron-scale dominate the dynamics. The reconnection rate $\left\langle E_{z}\right\rangle$ from our simulation is with a value as high as $\sim 0.6 B_{0} V_{A, 0} / c$ at the peak, much larger than the maximum rate of $0.1-0.2 B_{0} V_{A, 0} / c$ in anti-parallel Hallreconnection simulations ${ }^{12}$. Note that the maximum rate for Hall-reconnections with guide field is generally lower than the $0.1-0.2 B_{0} V_{A, 0} / c$ value.

How does the drag produce the electron-scale magnetic reconnection during Buneman instability? Previously ${ }^{51}$ we found that when $\Xi_{e}=0$, the Ohm's Law (Eq. 2) can be split 

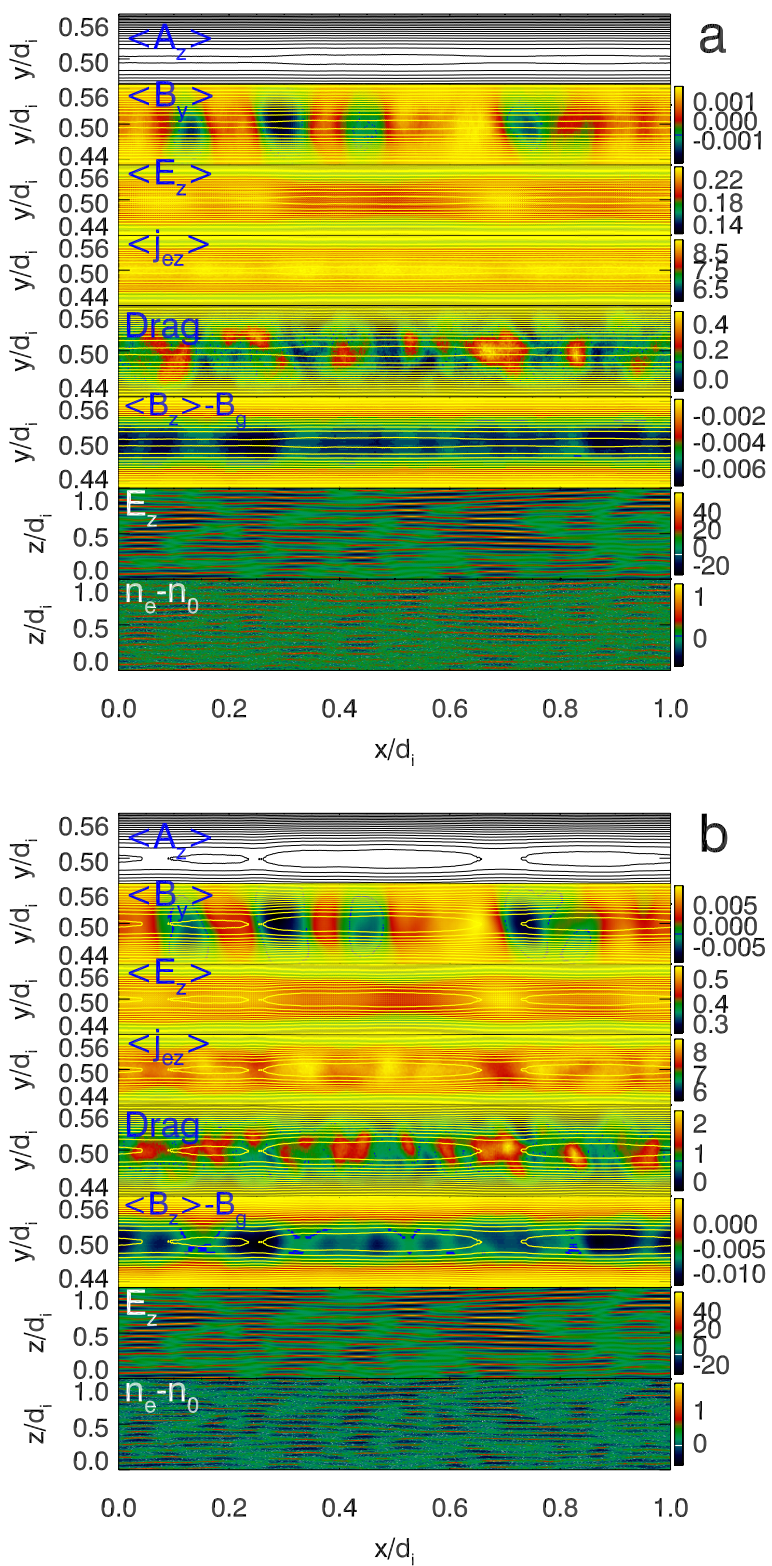

FIG. 1. (a): Quantities at $\omega_{p e, 0} t=48$ in the current sheet simulation when the Buneman instability occurs. (b): The same quantities as in (a) but at $\omega_{p e, 0} t=60$ when the Buneman instability reaches its peak. $\left\langle A_{z}\right\rangle$ is the z-component of the magnetic field vector potential. $E_{z}$ and electron density fluctuations $n_{e}-n_{0}$ are shown in the mid-plane x-z of the current sheet. 


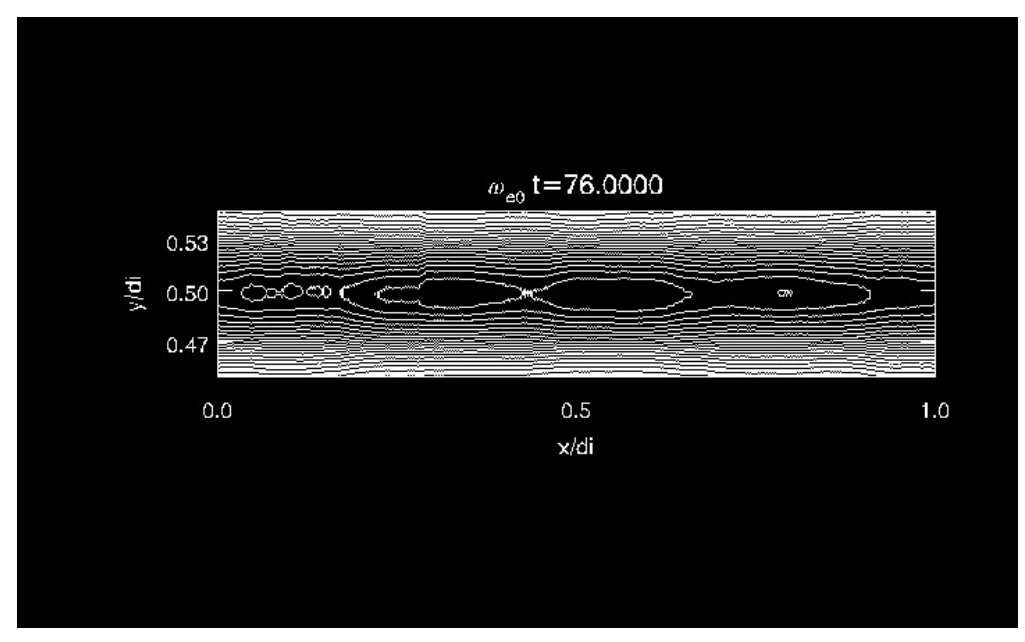

FIG. 2. The z-component of magnetic field vector potential $\left\langle A_{z}\right\rangle$ shows clear magnetic field line merging on the electron scale with amplitude $\left\langle A_{z}\right\rangle$ varying from 0 to 0.5 (Multimedia view).

into two equations around the time of the saturation of Buneman instability:

$$
\begin{gathered}
\left\langle E_{z}\right\rangle=-\frac{m_{e}}{e} \partial_{t}\left\langle U_{e z}\right\rangle+\left\langle D_{e z}\right\rangle \\
E_{z}^{w v}=E_{z}-\left\langle E_{z}\right\rangle=-\frac{m_{e}}{e} U_{e z} \partial_{z} U_{e z}-\frac{1}{e\left\langle n_{e}\right\rangle} \partial_{z} P_{e z z}
\end{gathered}
$$

where $E_{z}^{w v}$ is the Buneman instability generated coherent electric field, $\left\langle E_{z}\right\rangle$ is the mean electric field, and $E_{z}=E_{z}^{w v}+\left\langle E_{z}\right\rangle$. Buneman instability produces electron holes along $z$ near the mid-plane of the current sheets. The $z$-averaged $\left\langle E_{z}\right\rangle$ cancels out local effects of the electron holes since $\left\langle E_{z}^{w v}\right\rangle=0$. Eq. (3) establishes the relation between the reconnection rate and the drag while the average of Eq.(4) along z-direction indicates that the dissipation of kinetic energy by drag is converted into the parallel electron heating $\triangle\left\langle P_{e z z}\right\rangle=\triangle\left\langle m_{e} n_{e} U_{e z}^{2}\right\rangle / 2$ by the electron holes ${ }^{71}$. The inductive electric field is related to the magnetic vector potential by $\left\langle E_{z}\right\rangle=-\partial_{t}\left\langle A_{z}\right\rangle / c$ using the Coulomb gauge.

The wave couplings during the Buneman instability form wavepackets and cause the drag to become non-uniform within the current sheet and such non-uniformity determines the topology of the magnetic field. The spatial scale of wavepacket is determined by the uncertainty principle $\delta k \delta x \sim 2 \pi$, where $\delta k$ and $\delta x$ are the width of the wave number and the spatial size of the wave packet respectively. We approximate $\delta x \sim V_{g} \delta t$ and $\delta t \sim \omega_{p e}^{-1}$, where $V_{g} \sim d \omega / d t$. Using the $\omega-k$ relation for Buneman instability, we obtain $\delta k \sim k_{\text {fast }}$, $k_{f a s t}$ is the wave number of the fastest growing mode and satisfies $k_{f a s t}=\omega_{p e} / v_{d}$. In Fig. 1 , both the reconnection electric field and the drag appear "clumpy" in the x-direction and 
form "wave-packets" with scale $\sim 0.1 d_{i}=0.1 \sqrt{m_{i} / m_{e}} d_{e}=d_{e}$ for $m_{i} / m_{e}=100$, close to the wavelength of Buneman instability. Combining the relation $\left\langle E_{z}\right\rangle=-\partial_{t}\left\langle A_{z}\right\rangle / c$ and the Ampère's law, we obtain the equation for $\left\langle A_{z}\right\rangle$ around the mid-plane of the current sheet:

$$
\partial^{2}\left\langle A_{z}\right\rangle-\frac{1}{c^{2}} \partial_{t}^{2}\left\langle A_{z}\right\rangle+\frac{4 \pi}{c}\left\langle j_{e z}\right\rangle=0
$$

where $\partial^{2} \equiv \partial_{x}^{2}+\partial_{y}^{2}$, and we have neglected the contribution from $j_{e x}$ which is much smaller than $j_{e z}$. Eq.5 is a standard d'Alembert equation. The near field solution is

$$
\left\langle A_{z}(\mathbf{x})\right\rangle=\frac{1}{c} \int \frac{\left\langle j_{e z}\left(\mathbf{x}^{\prime}, t-r / c\right)\right\rangle}{r} d x d y
$$

where $\mathbf{r}=\mathbf{x}^{\prime}-\mathbf{x}$. The magnetic topology is determined by the inhomogeneity of the current sheet which is coupled to the drag by

$$
\frac{1}{c} \partial_{t}\left\langle A_{z}(\mathbf{x})\right\rangle=\frac{m_{e}}{e} \partial_{t}\left\langle j_{e z} / n_{e}\right\rangle-D_{e z}
$$

The magnetic vector potential is the near field solution of d'Alembert equation and the magnetic field is a quasi-static field, and its change in the xy plane describes the change of magnetic field topology $\left\langle B_{x}\right\rangle=-\partial_{y}\left\langle A_{z}\right\rangle$ and $\left\langle B_{y}\right\rangle=-\partial_{x}\left\langle A_{z}\right\rangle$. Using Eq.(7), we estimate how the electron-scale magnetic reconnection is determined by the inhomogeneity of the drag. From Eq.(7) we have $\gamma\left\langle A_{z}(\mathbf{x})\right\rangle / c \sim 4 \pi e \gamma \triangle\left\langle j_{e z}\right\rangle / \omega_{p e}^{2}-D_{e z}$, where $\gamma \sim \omega_{p e}$ is the growth rate of Buneman instability. Then we have $\left\langle A_{z}(\mathbf{x})\right\rangle \sim 4 \pi e \triangle\left\langle j_{e z}\right\rangle d_{e} / \omega_{p e}-D_{e z} d_{e}$, where $d_{e}$ is the electron inertial length $c / \omega_{p e}$. Thus $\left\langle B_{y}\right\rangle \sim\left(4 \pi e \triangle\left\langle j_{e z}\right\rangle / \omega_{p e}-D_{e z}\right) d_{e} / \triangle_{x}$, where $\triangle_{x}$ is the spatial scale of inhomogeneity of drag. It is clear that the spatial scale of the inhomogeneous turbulence drag is an important factor that determines the electron-scale magnetic reconnection.

It should be noted that the electron-scale magnetic reconnection is not necessary for the fast dissipation of magnetic energy during Buneman instability. The topology change of the field lines in Buneman instability requires the inhomogeneity of drag. The inhomogeneity is caused by wave couplings that break the symmetry of magnetic potential. To illustrate this point, we conduct a 2D Buneman instability simulation in the yz plane so that no electronscale magnetic reconnection can develop. Fig. 3 shows the electric field $E_{z}$ at $\omega_{p e, 0} t=60$. The intense localized bi-polar electric fields, i.e. electron holes form along the z-direction and the drag produced by these electron holes can dissipate the kinetic energy and the associated magnetic energy. At the same time, electron heating is produced in directions 


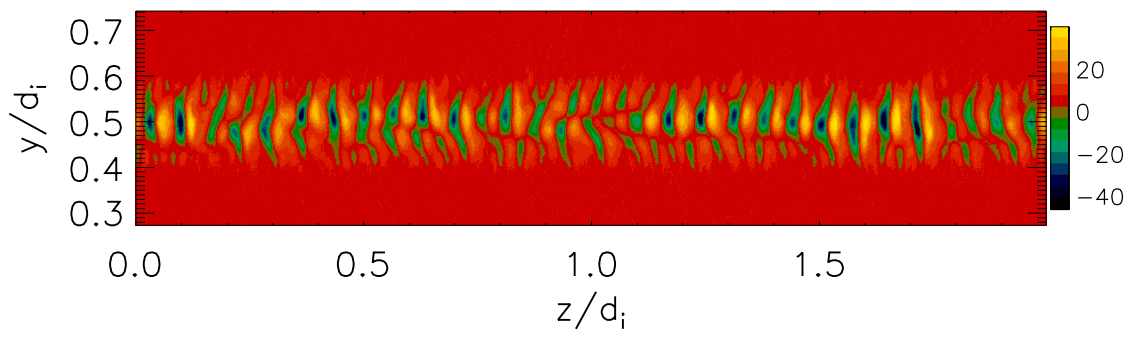

FIG. 3. $E_{z} / E_{0}$ in the 2 D Buneman instability simulation at $\omega_{p e, 0} t=60$, when electron holes are developed.

parallel and perpendicular to the magnetic field. The time evolutions of electron kinetic, magnetic energy and pressure are shown in Fig. 4, which are nearly identical to the evolutions of these quantities averaged over the x-direction in the 3D Buneman simulation as we found previously $^{51}$. It is clear that the inductive electric field is produced due to the fast dissipation of magnetic field (Fig. 5) whose time evolution is similar to that in the 3D simulation and the peak dissipation rate is $\sim 0.6 B_{0} V_{A 0} / c$. In Fig. $5, B_{y}$ generated by the Buneman instability oscillates on spatial scale $\sim 0.1 d_{i}$ similar to the wavelength of Buneman turbulence along $\mathrm{z}$ in the $2 \mathrm{D}$ simulation. The mean value of $\left\langle B_{y}\right\rangle$ is $\sim 10^{-6} \sim 0$, this confirms that no electronscale magnetic reconnection develops in the 2D simulation. For comparison, the evolution of $B_{y}$ along $\mathrm{z}$ at the same time in the 3D Buneman simulation is also shown. $B_{y}$ obviously deviates from zero. Beside the fast oscillations with spatial scale $0.1 d_{i}$ along z caused by the waves generated by the Buneman instability, a new feature is the long spatial scale variation caused by the wave-wave coupling of Buneman instability which leads to the nonzero $\left\langle B_{y}\right\rangle$. The value of $\left\langle B_{y}\right\rangle$ is 0.01 which is consistent with the value shown in Fig 1 for 3D Buneman 


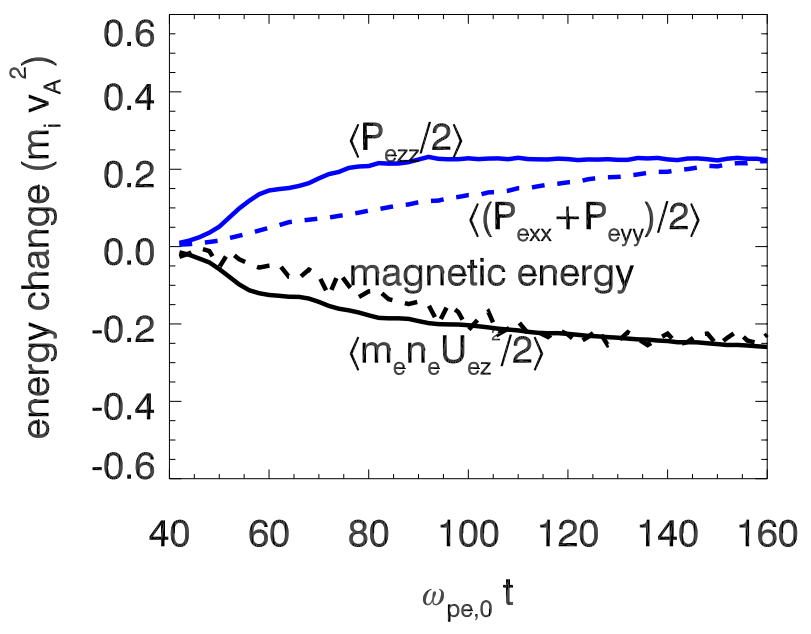

FIG. 4. The time evolution of energy change in 2D Buneman instability simulation. Black solid: kinetic energy of the electron beam; black dashed line: magnetic energy. Blue solid line: electron parallel thermal energy; blue dashed line: perpendicular thermal energy.

instability. This difference clearly demonstrates that $B_{y}$ is caused by the inhomogeneity of the drag.

To summarize, AR has two different but connected effects. First, it dissipates the magnetic energy and produces a mean inductive electric field; Second, the inhomogeneity of drag causes inhomogeneous magnetic energy dissipation which breaks the local symmetry of the magnetic field potential in the current sheet and leads to electron-scale magnetic reconnection. The electron-scale reconnection rate is significantly higher than Hall-reconnection rate. With this newly gained insight of Bumenan instability induced AR, we can look into the role of AR driven in ion-scale magnetic reconnection.

\section{How does Anomalous Resistivity Accelerate Magnetic Reconnection?}

Since the Buneman instability grows along the current sheet perpendicular to the reconnection plane, the instability cannot develop in 2D reconnection simulations. Comparing 

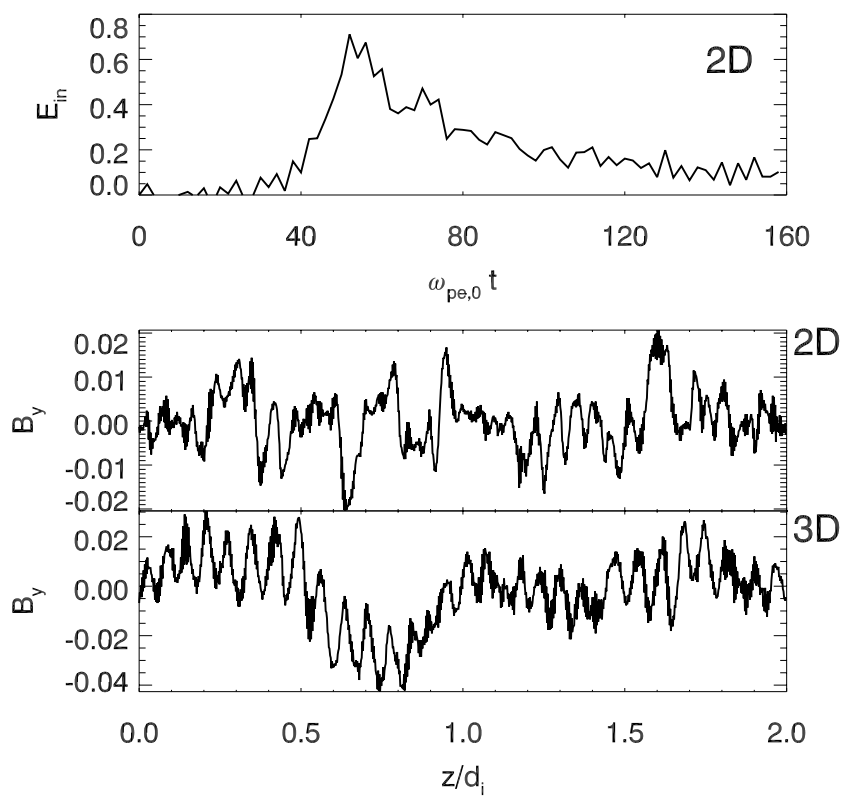

FIG. 5. Top panel: The time evolution of inductive electric field $\left\langle E_{z}\right\rangle$ in the 2D Buneman instability simulation. Bottom panel: The $B_{y}$ generated along $\mathrm{z}$ at $\omega_{p e, 0}=60$ in the $2 \mathrm{D}$ and $3 \mathrm{D}$ Buneman simulations.

our 3D and 2D simulations allows clear demonstration of the effects of turbulence generated by the Buneman instability. As we have shown previously, in the 3D magnetic reconnection simulation, turbulence driven by an ES Buneman instability and an EM electron velocity shear instability makes the magnetic reconnection significantly faster than the non-turbulent $2 \mathrm{D}$ reconnection ${ }^{37}$. The Buneman instability produces electron holes in the early stage for a brief period around $\Omega_{i 0} t \sim 0.4\left(\omega_{p e, 0} t=80\right)$ and the electron velocity shear instability starts to widen the current sheet and entails the filamentary structures at later stage. Close examination of Fig.4 in Che et al. $(2011)^{37}$ reveals that before the onset of Buneman instability, the reconnection electric field $\left\langle E_{z}\right\rangle$ at the $\mathrm{x}$-line (reconnection is in the xy plane) is fully supported by the electron inertia. As the Buneman instability is triggered at $\Omega_{i 0} t=3$ $\left(\omega_{p e, 0} t=600\right)$ when the electron drift is about $6 V_{A 0} \sim 2 v_{t e}$, the increasing drag reduces the electron acceleration, and eventually the drag becomes comparable to the electron inertia at $\Omega_{i 0} t=3.2\left(\omega_{p e, 0} t=640\right)$ and lasts to $\Omega_{i 0} t=3.4\left(\omega_{p e, 0} t=680\right)$. During this period the 

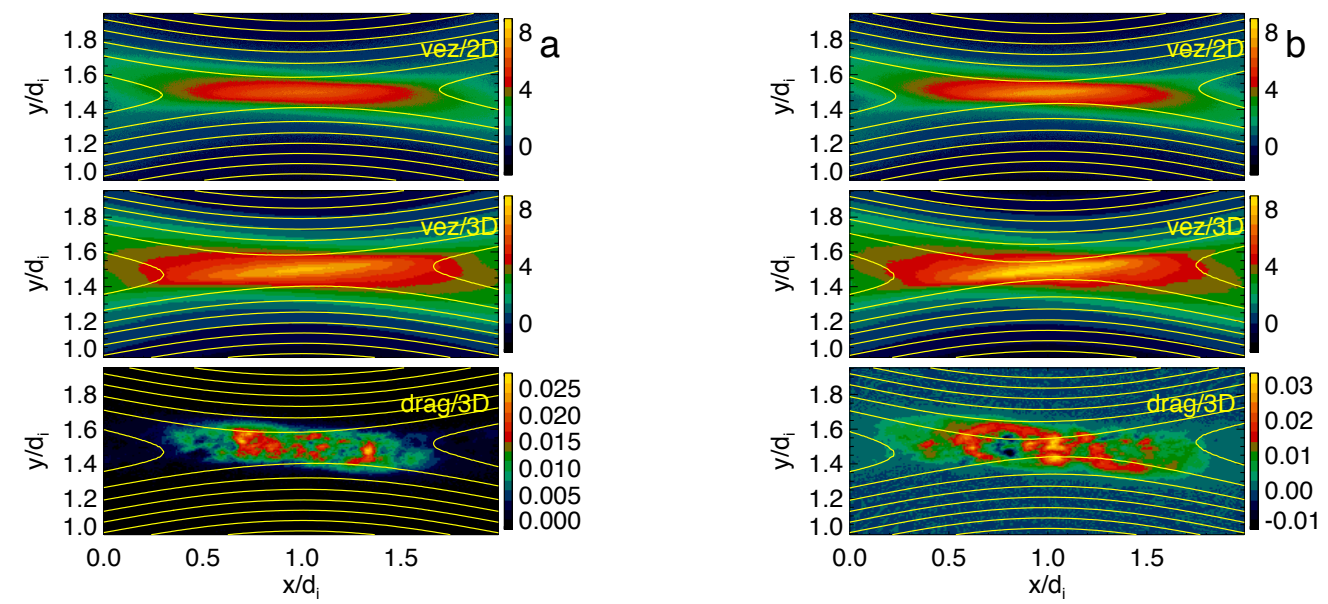

FIG. 6. The top two panels in $\mathbf{a} \& \mathbf{b}$ show the magnitude of electron velocity $V_{e z}$ in $3 \mathrm{D}$ and 2D magnetic reconnection, and the bottom panels show the drag $D_{e z}$. a: the onset of Buneman instability at $\Omega_{i 0} t=3\left(\omega_{p e, 0} t=600\right)$; b: the saturation of Buneman instability at $\Omega_{i 0} t=3.2$ $\left(\omega_{p e, 0} t=640\right)$. The solid lines represent the contour of magnetic vector potential $\left\langle A_{z}\right\rangle$.

Buneman instability becomes saturated. Then the Buneman instability starts to decay while the electron velocity shear instability starts to grow and surpasses the Buneman instability at $\Omega_{i 0} t \sim 3.8$. Then the induced whistler wave turbulence becomes dominant. After that the repeating occurrences of electron velocity shear instability continuously maintain the fast reconnection.

To understand how drag/AR accelerate magnetic reconnection, we need to answer two separate questions: 1) How does the drag break magnetic field lines around the x-point? and 2) can the drag affect the dynamics in the IDR?

In the preceding sections we have shown that $\mathrm{AR}$ can dissipate magnetic energy, produce inductive electric field and break magnetic field lines by its inhomogeneity in electron-scale thin current sheet. Here we show that the field-lines are also broken due to the inhomogeneity of the drag in the vicinity of the x-line to accelerate magnetic reconnection.

In Fig. 6 we show the electron velocity $V_{e z}$ in the $2 \mathrm{D}$ reconnection simulation and $\left\langle V_{e z}\right\rangle$ 
in the $3 \mathrm{D}$ reconnection simulation at $\Omega_{i 0} t=3\left(\omega_{p e, 0} t=600\right)$ when Buneman instability is triggered, and at $\Omega_{i 0} t=3.2\left(\omega_{p e, 0} t=640\right)$ when it reaches its saturation. The saturation lasts to $\Omega_{i 0} t=3.4\left(\omega_{p e, 0} t=680\right)$ when the drag becomes comparable to the inertia as we have shown previously ${ }^{37}$. The electron velocity $V_{e z}$ peaks around the x-point and exceeds the threshold $v_{t e}$, triggering Buneman instability. As a result, intensive drag is produced in the thin current layer around the x-point. We show the $\operatorname{drag} D_{e z}$ at $\Omega_{i 0} t=3,3.2$ in the xy plane in Fig. 6. Clearly the drag is spatially clumpy. While the drag is produced by Buneman instability at the vicinity of the x-point, the plasma outflow brings the inhomogeneous drag away from the x-point into the IDR, as illustrated by the expansion of the turbulent current sheet in Fig. 6. It should be noted that the AR induced dissipation reduces the acceleration of electron beams, but the electron beams are still continuously accelerated by the enhanced reconnection electric field and maintain the continuous growth of Buneman instability. Both the width and length of the current layer in the reconnection plane are larger in the 3D than in the $2 \mathrm{D}$ simulation, implying that the plasma heating produced by AR (drag) could play a similar role as collisional resistivity.

As the drag breaks the field lines at the x-point, the non-zero $B_{y}$ with opposite sign at the two sides of the x-point is produced, causing the null-point to shift in a stochastic manner in the 3D magnetic reconnection. In Fig.7, we show $E_{z}$ at $\Omega_{i 0} t=3.2$ in the yz plane at $x=d_{i}$, i.e. the location of the $\mathrm{x}$-point in the non-turbulent $2 \mathrm{D}$ reconnection. Bi-polar localized intense electric fields or electron holes form along z due to the Buneman instability near the x-point. In the bottom panel, we show $B_{y}(z)$ at the fiducial $2 \mathrm{D}$ x-point at times of $\Omega_{i 0} t=2.5$ (blue line) prior to the onset of the Buneman instability, and $\Omega_{i 0} t=3.2$ (black line) when the Buneman instability is near the peak. Large deviation of $B_{y}$ from zero is similar to $B_{y}$ produced in the 3D Buneman instability simulation shown in Fig.5. The deviation of $B_{y}$ from zero averaged over $\mathrm{z}$ is about 0.01 at $\Omega_{i 0} t=3.2$ while the mean value of $B_{y}$ is approximately zero at $\Omega_{i 0} t=2.5$. This stochastic shift of the x-point causes the EDR to become broader.

We further examine how the Buneman instability enhances the reconnection electric field $\left\langle E_{z}\right\rangle$. Fig. 8 shows $\left\langle E_{z}\right\rangle$ in the xy plane at $\Omega_{i} t=3.2\left(\omega_{p e, 0} t=640\right)$ in both the 3D and 2D simulations. Significant differences are found: 1) The reconnection electric field in the 3D simulation is concentrated in a region centered at the $\mathrm{x}$-point and extends in the $\mathrm{x}$-direction. On the other hand the reconnection electric field is more diffused in the $2 \mathrm{D}$ simulation, 


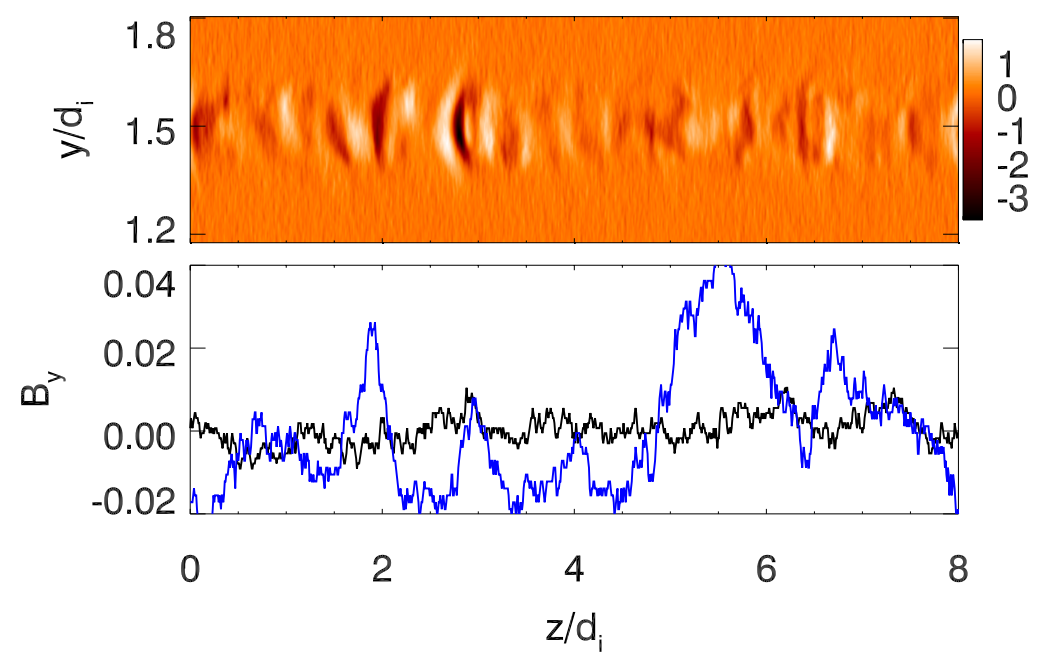

FIG. 7. Top panel: $E_{z}$ in yz plane at $\Omega_{i 0} t=3.2$ in $3 \mathrm{D}$ magnetic reconnection simulation. Bottom Panel: The evolution of $B_{y}$ along z at $\Omega_{i 0} t=2.5$ (black line) prior to the trigger of Buneman instability and $\Omega_{i 0} t=3.2$ (blue line) near the peak of Buneman instability in 3D magnetic reconnection simulation.

indicating that in $3 \mathrm{D}$ the reconnection rate is much higher in the vicinity of the $\mathrm{x}$-point as well as in the outflow where the drag is carried out than in regions where AR is small. Away from the x-point the electric field is similar to the mean field in the 2D simulation; 2) In the 3D simulation, the profile of $\left\langle E_{z}\right\rangle$ at the x-point resembles a "wavepacket". The spatial scale $\sim 0.1-0.2 d_{i}$ is consistent with the Buneman "wavepackets" in the current sheet simulation (c.f. Fig. 1) - implying that the reconnection is mediated by the inhomogeneity of the drag in the thin current sheet, and electron-scale magnetic reconnections occur around the x-line.

An important feature of the electron-scale magnetic reconnection is the enhancement of $\left\langle B_{y}\right\rangle$ by the inhomogeneous drag (Fig.6). We show $\left\langle B_{y}\right\rangle$ at $\Omega_{i 0} t=3.2$ in both $2 \mathrm{D}$ and $3 \mathrm{D}$ magnetic reconnections in Fig. 9. $\left\langle B_{y}\right\rangle$ is increased by $\sim 0.01$ in the area extending from the EDR to the IDR where $B_{y}^{2 D} \sim 0.04$ (taken at $x=1.6 d_{i}$ and $y=1.5$ in the $2 \mathrm{D}$ reconnection), or $\sim 25 \%$ enhancement in $3 \mathrm{D}$ than in $2 \mathrm{D}$. The enhancement of $\left\langle B_{y}\right\rangle$ is asymmetric around 


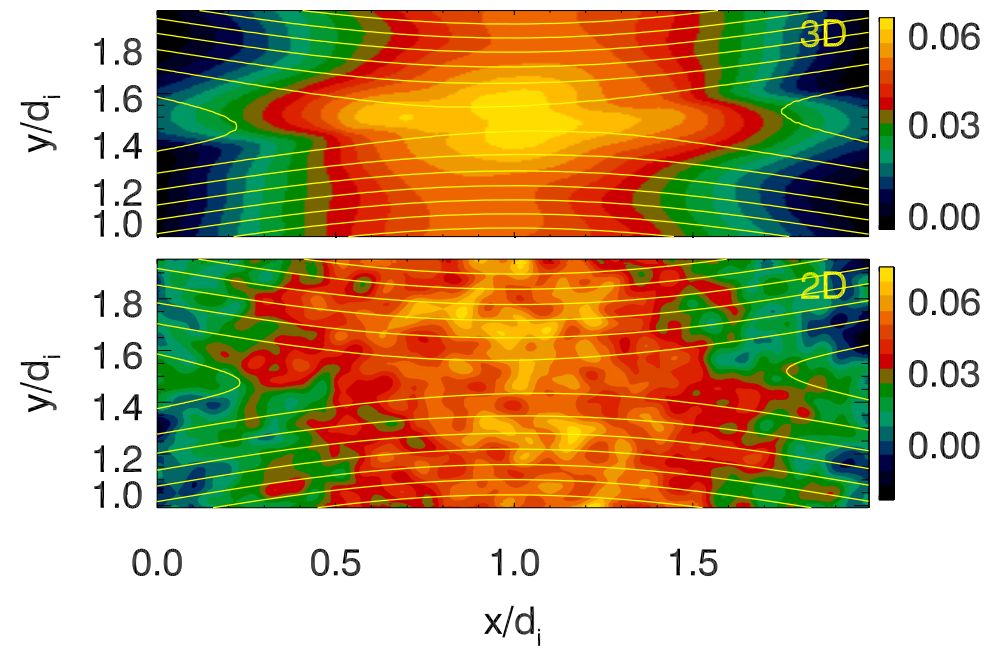

FIG. 8. The reconnection electric field $\left\langle E_{z}\right\rangle$ generated in $3 \mathrm{D}$ and $E_{z}$ in 2D magnetic reconnection at the saturation of Buneman instability $\Omega_{i} t=3.2\left(\omega_{p e, 0} t=640\right)$. The solid lines are the corresponding magnetic field lines.

the $\mathrm{x}$-point - this is due to the Hall-effect which generates an asymmetric quadratic electron current density in guide field magnetic reconnection ${ }^{19,30}$.

It can be shown that the enhancement of $B_{y}$ (denoted as $\left\langle B_{y}^{\text {bun }}\right\rangle$ ) in the IDR is related to the drag at the x-point in a rather simple way. In $\S$ II B we found $\left\langle B_{y}^{b u n}\right\rangle \sim\left(4 \pi e \triangle\left\langle j_{e z}\right\rangle / \omega_{p e}-\right.$ $\left.D_{e z}\right) d_{e} / \triangle_{x}$. The out-of-plane current $j_{e z}$ in the reconnection region is maintained by the reconnection electric field and the variation along $\mathrm{x}$ is small as seen in the simulation. On the other hand, the time for the plasma outflow to travel to the IDR is much shorter than the electron hole decay time, thus the drag is carried along with the outflow into the IDR. Neglecting the small decrease of $\left\langle j_{e z}\right\rangle$ we have $\left\langle B_{y}^{\text {bun }}\right\rangle \sim D_{e z} d_{e} / \triangle_{x}$. From Fig.6, $D_{e z} \sim 0.02$ and $\triangle_{x} \sim d_{e}$, we obtain $\left\langle B_{y}^{\text {bun }}\right\rangle \sim D_{e z} \sim 0.02$, which is consistent with the value in Fig.9.

The enhanced $B_{y}$ can increase the opening angle of local field lines at the x-line. However, in our simulation the increase of the angle $\triangle \theta$ in IDR is very small which can be estimated by $\triangle \theta \sim\left\langle B_{y}^{\text {bun }}\right\rangle \tan \theta / \tan \theta^{\prime} \sim \frac{1}{2}\left\langle B_{y}^{\text {bun }}\right\rangle \sin (2 \theta)<\sim \frac{1}{2}\left\langle B_{y}^{\text {bun }}\right\rangle \sim 0.01$ (about $0.5^{\circ}$ ), where 


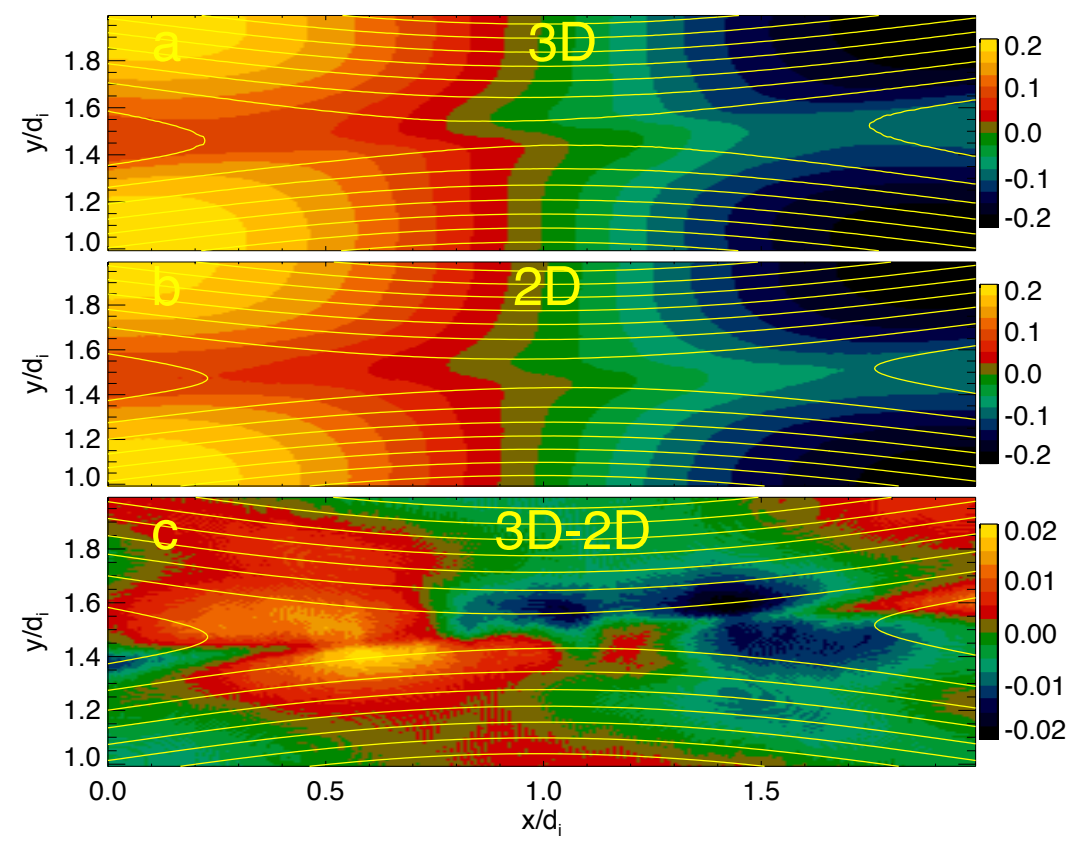

FIG. 9. $\left\langle B_{y}\right\rangle$ generated in 3D and $B_{y}$ in 2D magnetic reconnection at the peak of Buneman instability $\Omega_{i 0} t=3.2\left(\omega_{p e, 0} t=640\right)$. In bottom panel, the difference between $\left\langle B_{y}\right\rangle$ and $B_{y}$ is displayed. The contour of magnetic vector potential $\left\langle A_{z}\right\rangle$ is shown as solid lines.

$\tan \theta=B_{y} / B_{x}$

We now look at how the AR generated dissipation affect the magnetic reconnection. In Fig.10, we study the Poynting equation $\partial w / \partial t+\nabla \cdot \mathbf{S}+\mathbf{j}_{e} \cdot \mathbf{E}=0$, where $w=B^{2} / 4 \pi$ is the magnetic energy density. We consider the electromagnetic energy changes over the time from $\Omega_{i 0} t=3$ to $\Omega_{i 0} t=3.2\left(\triangle t=0.2 \Omega_{i 0}^{-1}\right)$, the period when the Buneman instability grows to saturation. Since $j_{e z} E_{z}$ contributes most to the heating $\mathbf{j}_{e} \cdot \mathbf{E}$, we neglect the $\mathrm{x}$ and y components. We can see that $j_{e z}^{2 D} E_{z}^{2 D}$ in the EDR of $2 \mathrm{D}$ reconnection is $\sim 0.5$ while $\left\langle j_{e z} E_{z}\right\rangle$ in the EDR of $3 \mathrm{D}$ reconnection is $\sim 0.8$. In $2 \mathrm{D} j_{e z}^{2 D} E_{z}^{2 D}$ corresponds to the magnetic energy that is converted into the kinetic energy of the electron beams near the $\mathrm{x}$-line due to the reconnection electric field acceleration. In $3 \mathrm{D}\left\langle j_{e z} E_{z}\right\rangle$ includes two parts according to Eq.(3) and (4), i.e. $\left\langle j_{e z}\right\rangle\left\langle E_{z}\right\rangle$ and $\left\langle j_{e z} E_{z}^{w v}\right\rangle$. The term $\left\langle j_{e z} E_{z}^{w v}\right\rangle=\left\langle\delta j_{e z} E_{z}^{w v}\right\rangle$ is the electron heating produced by electron holes. Given that $\left\langle j_{e z}\right\rangle\left\langle E_{z}\right\rangle=-m_{e}\left\langle j_{e z}\right\rangle \partial_{t}\left\langle U_{e z}\right\rangle / e+\left\langle j_{e z}\right\rangle D_{e z}$, the term $\left\langle j_{e z}\right\rangle\left\langle E_{z}\right\rangle$ includes the electron anomalous heating and the gain of the kinetic energy 

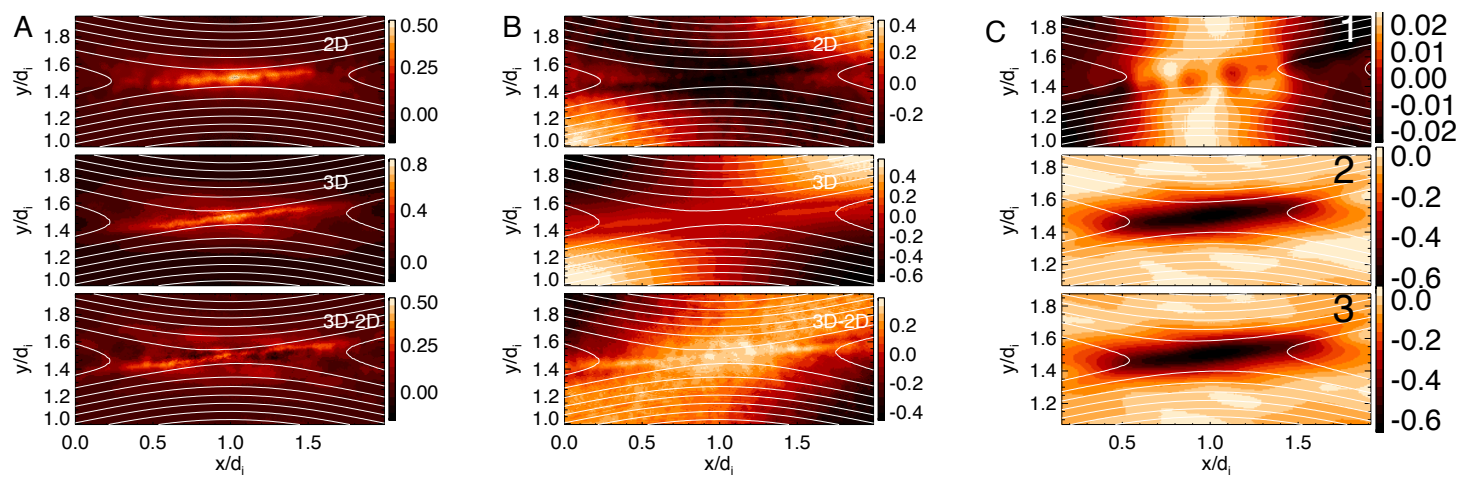

FIG. 10. Terms in the Poynting equation in the magnetic reconnection simulations. A. top panels: $\left\langle j_{e z} E_{z}\right\rangle$ in the $3 \mathrm{D}$ simulation and $j_{e z}^{2 D} E_{z}^{2 D}$ in the $2 \mathrm{D}$ simulation at $\Omega_{i 0} t=3.2$; bottom panel: $\left\langle j_{e z} E_{z}\right\rangle-j_{e z}^{2 D} E_{z}^{2 D}$. B. Top panels: the change of the magnetic energy density $\triangle\left(B_{x}^{2}+B_{y}^{2}+B_{z}^{2}\right) / 8 \pi$ in the $2 \mathrm{D}$ and $\triangle\left\langle\left(B_{x}^{2}+B_{y}^{2}+B_{z}^{2}\right) / 8 \pi\right\rangle$ in the $3 \mathrm{D}$ simulations between $\Omega_{i 0} t=3$ and $\Omega_{i 0} t=3.2$, divided by $0.2 \Omega_{i 0}^{-1}$. These are rough estimates of the average rate of change of the magnetic energy $\partial w / \partial t$; the bottom panel: The difference between $3 \mathrm{D}$ and $2 \mathrm{D}$ rates. C: The divergence of Poynting flux. 1) $\left.\left.\partial_{x} S_{x}, 2\right) \partial_{y} S_{y}, 3\right) \partial_{x} S_{x}+\partial_{y} S_{y}$.

of electron beams by acceleration. In Fig. 6, the velocities of the electron beams in both the $2 \mathrm{D}$ and $3 \mathrm{D}$ reconnection are roughly the same, meaning the same amount of magnetic energy is used to accelerate the electron beams in both cases, and therefore the AR induced electron heating can be estimated by $\left\langle j_{e z} E_{z}\right\rangle-j_{e z}^{2 D} E_{z}^{2 D}$ shown in Fig. $10 \mathrm{~A}$, and $\sim 30-50 \%$ of the magnetic energy turns into the thermal energy of electrons in the AR dominated phase of the reconnection.

We show the change of magnetic energy in Fig. 10B. The rate of magnetic energy loss $\partial w / \partial t$ near the $\mathrm{x}$-line in the $2 \mathrm{D}$ reconnection approximately matches $j_{e z}^{2 D} E_{z}^{2 D}$. However, in the $3 \mathrm{D}$ reconnection, $\partial w / \partial t$ in the EDR is close to zero-indicating that there should be an extra Poynting flux flowing into the EDR. The difference between the magnetic energy change rates in the $3 \mathrm{D}$ and $2 \mathrm{D}$ reconnections further illustrates that magnetic energy is flowing into the EDR in the 3D reconnection. Obviously the Buneman instability at the $\mathrm{x}$-point is responsible for such energy transfer. To illustrate the magnetic energy transfer, we examine the divergence of Poynting flux. Consistent with what we see in the 2D simulation $\partial_{t} w+j_{e z}^{2 D} E_{z}^{2 D} \sim 0$, the divergence of Poynting flux in the EDR is close to zero (not shown here). The divergence of Poynting flux in the $3 \mathrm{D}$ reconnection is shown in Fig. 10C. We 
found the Poynting flux flowing into EDR in y direction $(c / 4 \pi) \partial_{y}\left\langle E_{z} B_{x}\right\rangle$ (panel C2) balances the dissipation which accounts for $\sim 40 \%$ of the released magnetic energy in the EDR, in other words the AR enhanced $E_{z}$ is responsible for the magnetic energy transfer into the EDR to accelerate the reconnection. Compared to the in-flow of magnetic energy, 10\% of the released magnetic energy is brought out to the IDR by the enhanced $B_{y}$ through the $\mathrm{x}$-component of the Poynting flux $\sim-(c / 4 \pi) \partial_{y}\left\langle E_{z} B_{y}\right\rangle$ (panel C1).

\section{DISCUSSION AND CONCLUSIONS}

In this paper, we explore how Buneman instability induced AR/drag at the x-line accelerates 3D guide-field magnetic reconnection. With a 3D PIC simulation of Buneman instability in an electron scale current sheet we show that 1) drag $D_{e z}$ can dissipate magnetic energy stored in the current layer $j_{e z}$ through the dissipation of the kinetic energy of electron beams. This process is described by the Ohm's law $\left.\left\langle E_{z}\right\rangle=-\frac{m_{e}}{e} \partial_{t}\left\langle j_{e z} / n_{e}\right\rangle+D_{e z} ; 2\right)$ the coupling of waves leads to the formation of wavepackets, causing the drag $D_{e z}$ to become inhomogeneous along $\mathrm{x}$. As a result, the inductive electric field $\left\langle E_{z}\right\rangle$ is also a spatial function, which in turn leads to fast impulsive electron-scale magnetic reconnections as the inhomogeneous drag breaks the symmetry of the magnetic potential through $-\partial_{t}\left\langle A_{z}\right\rangle / c=\left\langle E_{z}\right\rangle$, and generates the perpendicular magnetic field $\left\langle B_{y}^{b u n}\right\rangle=-\partial_{x}\left\langle A_{z}\right\rangle$.

The electron-scale magnetic reconnection caused by AR is not affected by Hall-effect. The reconnection rate can reach as high as $0.6 B_{0} V_{A 0} / c$, and such a high rate has not been seen previously in simulations. The electron-scale magnetic reconnection however does not alter the overall energy conversion rates between kinetic, thermal and magnetic energy in the current sheet, as shown in a comparison between 2D and 3D Buneman instability simulations.

In ion-scale reconnection simulations, we demonstrate how AR affects magnetic reconnection. We were able to separate the effects of AR from other non-turbulent effects that accelerate magnetic reconnection by comparing the full $3 \mathrm{D}$ reconnection simulation with a benchmark 2D simulation in which Buneman instability does not develop. It should be noted that in the 3D ion-scale reconnection simulation, the Buneman instability developed at the $\mathrm{x}$-line is weaker than that in the electron-scale current sheet simulation. This is because 1) the initial electron drift in reconnection simulation is $4 V_{A 0}$ and the instability is triggered as 
the drift increased to $6 V_{A 0} \sim 2 v_{t e}$, much smaller than the initial electron drift $9 V_{A 0} \sim 3 v_{t e}$ in the current sheet simulation; 2) while the velocity of electron beams eventually reaches $9 V_{A 0}$ at later times of the simulation, the parallel electron thermal velocity also increases to $5 V_{A 0}$ due to the electron heating produced by the Buneman instability. As a result the electron holes and the inductive electric field produced in the $3 \mathrm{D}$ reconnection simulation are all much weaker than that in the Buneman instability simulation even though the 3D magnetic reconnection is still much faster than the corresponding $2 \mathrm{D}$ magnetic reconnection.

With these simulations, we have found the following: 1) The drag significantly increase the reconnection electric field at the x-point and in the reconnection out-flow region extending to the IDR; 2) The inhomogeneity of the drag can break the magnetic field lines to produce electron-scale magnetic reconnections at the x-line; 3) The drag can be brought out by the reconnection out-flow into the IDR, thus increases the size of the EDR; 4) The drag also increases the size of the EDR by stochastic shifts of the x-point; 5) The enhanced perpendicular magnetic field $B_{y}$ is increased by an average of $\sim 25 \%$ in our simulation; and 6) About $40 \%$ of the released magnetic energy is converted into the electron thermal energy by AR while $50 \%$ is converted into the kinetic energy of the electron beams through the acceleration by reconnection electric field. The enhanced magnetic energy dissipation is supported by a net Poynting flux in-flow. About $10 \%$ of the released magnetic energy is brought out by the enhanced Poynting flux out-flow.

We have shown that $B_{y}^{b u n}$ is related to the drag by $B_{y}^{b u n} \sim D_{e z} d_{e} / \triangle_{x}$, where $\triangle_{x}$ is the spatial scale of inhomogeneity of drag. Therefore only small $\triangle_{x}$ can lead to large $B_{y}^{b u n}$ (in our case $\left.\triangle_{x} \sim d_{e}\right)$ - implying perhaps only drag with electron scale inhomogeneity has the potential to significantly accelerate magnetic reconnection, probably faster than the Hall reconnection rate of $0.1 V_{A}$. This may explain why some PIC reconnection simulations with electron holes developed do not show one-to-one correspondence between reconnection rate and energy conversion ${ }^{73-75}$.

A group of electron beam instabilities commonly known as streaming instabilities which include Buneman instability, electron two-stream instability ${ }^{60}$ and lower hybrid instability ${ }^{62}$ etc, can all develop around the x-line and in the separatrix as the reconnection current sheets proceed to the electron inertial length. These electron electrostatic instabilities can form coherent structures such as electron holes and efficiently dissipate both of the kinetic and magnetic energy via AR. The results presented in this paper serve as a case study which 
should be useful for more general inquiries into the role of AR in magnetic reconnection.

The results presented in this paper are potentially observable in the magnetosphere. The MMS time resolution of $\sim \omega_{p e}^{-1}$ for high frequency waves ${ }^{76}$ can resolve the waves generated during the nonlinear phase of the Buneman instability, which lasts for $\omega_{p e, 0} t \sim 80$. Interesting enough, a spontaneous small-scale fast guide field reconnection produced by the twisted magnetic flux tube may have been observed by $\mathrm{MMS}^{77}$. Streaming instabilities and electron holes in EDRs of magnetic reconnections are also discovered by $\mathrm{MMS}^{32,54-58}$. With more observated events, the MMS mission is in a good position to observationally determine the role of turbulence in magnetic reconnection ${ }^{78,79}$.

\section{ACKNOWLEDGMENTS}

This research is supported by the NASA $M M S$ in association with NASA contract NNG04EB99C. The author thanks the entire $M M S$ project for the support, in particular the discussions with colleagues in the FPI team: J. Dorelli, W. Paterson, B. Giles, M. Goldstein, L. Avanov, B. Lavraud, M. Chandler, D. Gershman and C. Schiff, and the participants of 2016 1st MMS community Science Workshop in UCLA. The simulations and analysis were carried out at the NASA Advanced Supercomputing facility at the Ames Research Center and the National Energy Research Scientific Computing Center. The author thanks the anonymous referee for the critical and constructive comments that helps to improve the clarity of this manuscript. 


\section{REFERENCES}

${ }^{1}$ C. Robertson, S. W. H. Cowley, and J. W. Dungey, "Wave-particle interactions in a magnetic neutral sheet," Planetary and Space Science 29, 399-403 (1981).

${ }^{2} \mathrm{~J}$. Berchem and C. T. Russell, "The thickness of the magnetopause current layer - ISEE 1 and 2 observations," J. Geophys. Res. 87, 2108-2114 (1982).

${ }^{3}$ C. T. Russell, X.-W. Zhou, G. Le, P. H. Reiff, J. G. Luhmann, C. A. Cattell, and H. Kawano, "Field aligned currents in the high latitude, high altitude magnetosphere: POLAR initial results," Geophys. Res. Lett. 24, 1455-1458 (1997).

${ }^{4}$ D. E. Wendel and M. L. Adrian, "Current structure and nonideal behavior at magnetic null points in the turbulent magnetosheath," J. Geophys. Res. 118, 1571-1588 (2013).

${ }^{5}$ G. Le, H. Lhr, B. J. Anderson, R. J. Strangeway, C. T. Russell, H. Singer, J. A. Slavin, Y. Zhang, T. Huang, K. Bromund, P. J. Chi, G. Lu, D. Fischer, E. L. Kepko, H. K. Leinweber, W. Magnes, R. Nakamura, F. Plaschke, J. Park, J. Rauberg, C. Stolle, and R. B. Torbert, "Magnetopause erosion during the 17 march 2015 magnetic storm: Combined field-aligned currents, auroral oval, and magnetopause observations," Geophys. Res. Lett. 43, 2396-2404 (2016), 2016GL068257.

${ }^{6}$ H. Karimabadi, V. Roytershteyn, W. Daughton, and Y.-H. Liu, "Recent Evolution in the Theory of Magnetic Reconnection and Its Connection with Turbulence," Space Sci. Rev. 178, 307-323 (2013).

${ }^{7} \mathrm{~J}$. A. Klimchuk, "Key Aspects of Coronal Heating," in AAS/AGU Triennial Earth-Sun Summit, AAS/AGU Triennial Earth-Sun Summit, Vol. 1 (2015) p. 203.08.

${ }^{8}$ M. Velli, F. Pucci, F. Rappazzo, and A. Tenerani, "Models of coronal heating, turbulence and fast reconnection," Philosophical Transactions of the Royal Society of London Series A 373, 20140262-20140262 (2015).

${ }^{9}$ P. A. Sweet, "The Neutral Point Theory of Solar Flares," in IAU Symp. 6: Electromagnetic Phenomena in Cosmical Physics, edited by B. Lehnert (1958) pp. 123-134.

${ }^{10}$ B. U. Ö. Sonnerup, "Magnetic Field Reconnection," in Space Plasma Physics: The Study of Solar-System Plasmas. Volume 2 (1979) pp. 879-+.

${ }^{11}$ D. Biskamp, "Magnetic reconnection," Physics Reports 237, 179-247 (1994).

${ }^{12}$ J. Birn, J. F. Drake, M. A. Shay, B. N. Rogers, R. E. Denton, M. Hesse, M. Kuznetsova, Z. W. Ma, A. Bhattacharjee, A. Otto, and P. L. Pritchett, "Geospace Environmental Mod- 
eling (GEM) magnetic reconnection challenge," J. Geophys. Res. 106, 3715-3720 (2001).

${ }^{13} \mathrm{H}$. Che, Non-linear development of streaming instabilities in magnetic reconnection with a strong guide field, Ph.D. thesis, University of Maryland, College Park (2009).

${ }^{14}$ V. M. Vasyliunas, "Theoretical Models of Magnetic Field Line Merging, 1," Reviews of Geophysics and Space Physics 13, 303-+ (1975).

${ }^{15}$ D. A. Uzdensky and R. M. Kulsrud, "Physical origin of the quadrupole out-of-plane magnetic field in Hall-magnetohydrodynamic reconnection," Phys. Plasma 13, 062305-062305 (2006).

${ }^{16}$ J. F. Drake, M. A. Shay, and M. Swisdak, "The Hall fields and fast magnetic reconnection," Phys. Plasma 15, 042306 (2008).

${ }^{17}$ M. Hesse, K. Schindler, J. Birn, and M. Kuznetsova, "The diffusion region in collisionless magnetic reconnection," Phys. Plasma 6, 1781-1795 (1999).

${ }^{18}$ M. Hesse, M. Kuznetsova, and M. Hoshino, "The structure of the dissipation region for component reconnection: Particle simulations," Geophys. Res. Lett. 29, 4-1 (2002).

${ }^{19} \mathrm{~J}$. Birn and E. R. Priest, Reconnection of magnetic fields : magnetohydrodynamics and collisionless theory and observations (Reconnection of magnetic fields : magnetohydrodynamics and collisionless theory and observations / edited by J. Birn and E. R. Priest. Cambridge : Cambridge University Press, 2007. ISBN: 9780521854207 (hbk.), 2007).

${ }^{20}$ M. A. Shay and J. F. Drake, "The role of electron dissipation on the rate of collisionless magnetic reconnection," Geophys. Res. Lett. 25, 3759-3762 (1998).

${ }^{21}$ M. A. Shay, J. F. Drake, B. N. Rogers, and R. E. Denton, "The scaling of collisionless, magnetic reconnection for large systems," Geophys. Res. Lett. 26, 2163-2166 (1999).

${ }^{22}$ M. Øieroset, T. D. Phan, M. Fujimoto, R. P. Lin, and R. P. Lepping, "In situ detection of collisionless reconnection in the Earth's magnetotail," Nature 412, 414-417 (2001).

${ }^{23}$ Y. Ren, M. Yamada, S. Gerhardt, H. Ji, R. Kulsrud, and A. Kuritsyn, "Experimental Verification of the Hall Effect during Magnetic Reconnection in a Laboratory Plasma," Phys. Rev. Lett. 95, 055003-+ (2005).

${ }^{24}$ M. Yamada, Y. Ren, H. Ji, J. Breslau, S. Gerhardt, R. Kulsrud, and A. Kuritsyn, "Experimental study of two-fluid effects on magnetic reconnection in a laboratory plasma with variable collisionality," Phys. Plasma 13, 052119 (2006).

${ }^{25}$ Y. Ren, M. Yamada, H. Ji, S. Dorfman, S. P. Gerhardt, and R. Kulsrud, "Experimental study of the Hall effect and electron diffusion region during magnetic reconnection in a 
laboratory plasma," Physics of Plasmas 15, 082113 (2008).

${ }^{26}$ B. R. Dennis, A. G. Emslie, and H. S. Hudson, "Overview of the Volume," Space Sci. Rev. 159, 3-17 (2011).

${ }^{27}$ L. Fletcher, B. R. Dennis, H. S. Hudson, S. Krucker, K. Phillips, A. Veronig, M. Battaglia, L. Bone, A. Caspi, Q. Chen, P. Gallagher, P. T. Grigis, H. Ji, W. Liu, R. O. Milligan, and M. Temmer, "An Observational Overview of Solar Flares," Space Sci. Rev. 159, 19-106 (2011).

${ }^{28}$ H. Che, M. L. Goldstein, P. H. Diamond, and R. Z. Sagdeev, "How electron two-stream instability drives cyclic langmuir collapse and continuous coherent emission," Proceedings of the National Academy of Sciences 114, 1502-1507 (2017), http://www.pnas.org/content/114/7/1502.full.pdf.

${ }^{29}$ Y. Su, A. M. Veronig, G. D. Holman, B. R. Dennis, T. Wang, M. Temmer, and W. Gan, "Imaging coronal magnetic-field reconnection in a solar flare," Nature Physics 9, 489-493 (2013).

${ }^{30}$ T. D. Tharp, M. Yamada, H. Ji, E. Lawrence, S. Dorfman, C. E. Myers, and J. Yoo, "Quantitative Study of Guide-Field Effects on Hall Reconnection in a Laboratory Plasma," Phys. Rev. Lett. 109, 165002 (2012).

${ }^{31}$ M. V. Goldman, D. L. Newman, and G. Lapenta, "What Can We Learn about Magnetotail Reconnection from 2D PIC Harris-Sheet Simulations?" Space Sci. Rev. 199, 651-688 (2016).

${ }^{32}$ R. B. Torbert, J. L. Burch, B. L. Giles, D. Gershman, C. J. Pollock, J. Dorelli, L. Avanov, M. R. Argall, J. Shuster, R. J. Strangeway, C. T. Russell, R. E. Ergun, F. D. Wilder, K. Goodrich, H. A. Faith, C. J. Farrugia, P.-A. Lindqvist, T. Phan, Y. Khotyaintsev, T. E. Moore, G. Marklund, W. Daughton, W. Magnes, C. A. Kletzing, and S. Bounds, "Estimates of terms in Ohm's law during an encounter with an electron diffusion region," Geophys. Res. Lett. 43, 5918-5925 (2016).

${ }^{33}$ H. Ji, M. Yamada, S. Hsu, and R. Kulsrud, "Experimental Test of the Sweet-Parker Model of Magnetic Reconnection," Phys. Rev. Lett. 80, 3256-3259 (1998).

${ }^{34}$ R. M. Kulsrud, "Magnetic reconnection: Sweet-Parker versus Petschek," Earth Planet. Space 53, 417-422 (2001).

${ }^{35} \mathrm{~L}$. Comisso and A. Bhattacharjee, "On the value of the reconnection rate," Journal of Plasma Physics 82, 595820601 (2016). 
${ }^{36}$ M. I. Sitnov, V. G. Merkin, P. L. Pritchett, and M. Swisdak, "Distinctive features of internally driven magnetotail reconnection," Geophysical Research Letters , n/a-n/a (2017), 2017 GL072784.

${ }^{37}$ H. Che, J. F. Drake, and M. Swisdak, "A current filamentation mechanism for breaking magnetic field lines during reconnection," Nature 474, 184 (2011).

${ }^{38}$ L. Fletcher, "Ultra-violet footpoints as tracers of coronal magnetic connectivity and restructuring during a solar flare," Astronomy and Astrophysics 493, 241-250 (2009).

${ }^{39}$ J. Lin, N. A. Murphy, C. Shen, J. C. Raymond, K. K. Reeves, J. Zhong, N. Wu, and Y. Li, "Review on Current Sheets in CME Development: Theories and Observations," Space Sci. Rev. 194, 237-302 (2015).

${ }^{40}$ K. Papadopoulos, "A review of anomalous resistivity for the ionosphere." Reviews of Geophysics and Space Physics 15, 113-127 (1977).

${ }^{41} \mathrm{~A}$. Bhattacharjee and E. Hameiri, "Self-consistent dynamolike activity in turbulent plasmas," Phys. Rev. Lett. 57, 206-209 (1986).

${ }^{42}$ B. Coppi, G. Laval, and R. Pellat, "Dynamics of the Geomagnetic Tail," Phys. Rev. Lett. 16, 1207-1210 (1966).

${ }^{43}$ B. Coppi and A. B. Friedland, "Processes of Magnetic-Energy Conversion and Solar Flares," The Astrophysical Journal 169, 379 (1971).

${ }^{44}$ J. D. Huba, N. T. Gladd, and K. Papadopoulos, "The lower-hybrid-drift instability as a source of anomalous resistivity for magnetic field line reconnection," Geophys. Res. Lett. 4, 125-126 (1977).

${ }^{45}$ A. A. Galeev, "Reconnection in the magnetotail," Space Sci. Rev. 23, 411-425 (1979).

${ }^{46}$ P. K. Kaw, E. J. Valeo, and P. H. Rutherford, "Tearing modes in a plasma with magnetic braiding," Phys. Rev. Lett. 43, 1398-1401 (1979).

${ }^{47}$ A. A. Galeev and R. Z. Sagdeev, "Current instabilities and anomalous resistivity of plasma," in Basic Plasma Physics: Selected Chapters, Handbook of Plasma Physics, Volume II, edited by A. A. Galeev and R. N. Sudan (1984) pp. 271-303.

${ }^{48}$ E. N. Parker, "Anomalous resistivity and the evolution of magnetic field topology," The Astrophysical Journal 414, 389-398 (1993).

${ }^{49}$ Z. B. Guo, P. H. Diamond, and X. G. Wang, "Magnetic Reconnection, Helicity Dynamics, and Hyper-diffusion," The Astrophysical Journal 757, 173 (2012).

${ }^{50}$ S. J. Schwartz, E. G. Zweibel, and M. Goldman, "Microphysics in Astrophysical Plasmas," 
Space Sci. Rev. 178, 81-99 (2013).

${ }^{51} \mathrm{H}$. Che, "Two-fluid description of wave-particle interactions in strong Buneman turbulence," 21, 062305 (2014).

${ }^{52}$ P. A. Muñoz and J. Büchner, "Non-Maxwellian electron distribution functions due to selfgenerated turbulence in collisionless guide-field reconnection," Phys. Plasma 23, 102103 (2016).

${ }^{53}$ P. A. Muñoz, J. Büchner, and P. Kilian, "Turbulent transport in 2D collisionless guide field reconnection," Physics of Plasmas 24, 022104 (2017).

${ }^{54}$ Y. V. Khotyaintsev, D. B. Graham, C. Norgren, E. Eriksson, W. Li, A. Johlander, A. Vaivads, M. André, P. L. Pritchett, A. Retinò, T. D. Phan, R. E. Ergun, K. Goodrich, P.-A. Lindqvist, G. T. Marklund, O. Le Contel, F. Plaschke, W. Magnes, R. J. Strangeway, C. T. Russell, H. Vaith, M. R. Argall, C. A. Kletzing, R. Nakamura, R. B. Torbert, W. R. Paterson, D. J. Gershman, J. C. Dorelli, L. A. Avanov, B. Lavraud, Y. Saito, B. L. Giles, C. J. Pollock, D. L. Turner, J. D. Blake, J. F. Fennell, A. Jaynes, B. H. Mauk, and J. L. Burch, "Electron jet of asymmetric reconnection," Geophys. Res. Lett. 43, 5571-5580 (2016).

${ }^{55}$ F. D. Wilder, R. E. Ergun, K. A. Goodrich, M. V. Goldman, D. L. Newman, D. M. Malaspina, A. N. Jaynes, S. J. Schwartz, K. J. Trattner, J. L. Burch, M. R. Argall, R. B. Torbert, P.-A. Lindqvist, G. Marklund, O. Le Contel, L. Mirioni, Y. V. Khotyaintsev, R. J. Strangeway, C. T. Russell, C. J. Pollock, B. L. Giles, F. Plaschke, W. Magnes, S. Eriksson, J. E. Stawarz, A. P. Sturner, and J. C. Holmes, "Observations of whistler mode waves with nonlinear parallel electric fields near the dayside magnetic reconnection separatrix by the Magnetospheric Multiscale mission," Geophys. Res. Lett. 43, 5909-5917 (2016).

${ }^{56}$ R. Nakamura, V. A. Sergeev, W. Baumjohann, F. Plaschke, W. Magnes, D. Fischer, A. Varsani, D. Schmid, T. K. M. Nakamura, C. T. Russell, R. J. Strangeway, H. K. Leinweber, G. Le, K. R. Bromund, C. J. Pollock, B. L. Giles, J. C. Dorelli, D. J. Gershman, W. Paterson, L. A. Avanov, S. A. Fuselier, K. Genestreti, J. L. Burch, R. B. Torbert, M. Chutter, M. R. Argall, B. J. Anderson, P.-A. Lindqvist, G. T. Marklund, Y. V. Khotyaintsev, B. H. Mauk, I. J. Cohen, D. N. Baker, A. N. Jaynes, R. E. Ergun, H. J. Singer, J. A. Slavin, E. L. Kepko, T. E. Moore, B. Lavraud, V. Coffey, and Y. Saito, "Transient, small-scale field-aligned currents in the plasma sheet boundary layer during storm time substorms," Geophys. Res. Lett. 43, 4841-4849 (2016). 
${ }^{57}$ R. E. Ergun, J. C. Holmes, K. A. Goodrich, F. D. Wilder, J. E. Stawarz, S. Eriksson, D. L. Newman, S. J. Schwartz, M. V. Goldman, A. P. Sturner, D. M. Malaspina, M. E. Usanova, R. B. Torbert, M. Argall, P.-A. Lindqvist, Y. Khotyaintsev, J. L. Burch, R. J. Strangeway, C. T. Russell, C. J. Pollock, B. L. Giles, J. J. C. Dorelli, L. Avanov, M. Hesse, L. J. Chen, B. Lavraud, O. Le Contel, A. Retino, T. D. Phan, J. P. Eastwood, M. Oieroset, J. Drake, M. A. Shay, P. A. Cassak, R. Nakamura, M. Zhou, M. Ashour-Abdalla, and M. Andr, "Magnetospheric multiscale observations of large-amplitude, parallel, electrostatic waves associated with magnetic reconnection at the magnetopause," Geophys. Res. Lett. 43, 5626-5634 (2016), 2016GL068992.

${ }^{58}$ D. B. Graham, Y. V. Khotyaintsev, A. Vaivads, and M. André, "Electrostatic solitary waves and electrostatic waves at the magnetopause," J. Geophys. Res. 121, 3069-3092 (2016).

${ }^{59}$ I. H. Hutchinson, "Electron holes in phase space: What they are and why they matter," Phys. Plasma 24, 055601 (2017).

${ }^{60}$ H. Che, J. F. Drake, M. Swisdak, and P. H. Yoon, "Nonlinear Development of Streaming Instabilities in Strongly Magnetized Plasma," Phys. Rev. Lett. 102, 145004-+ (2009).

${ }^{61}$ J. F. Drake, M. Swisdak, C. Cattell, M. A. Shay, B. N. Rogers, and A. Zeiler, "Formation of Electron Holes and Particle Energization During Magnetic Reconnection," Science 299, 873-877 (2003).

${ }^{62}$ H. Che, J. F. Drake, M. Swisdak, and P. H. Yoon, "Electron holes and heating in the reconnection dissipation region," Geophys. Res. Lett. 37, 11105-+ (2010).

${ }^{63}$ A. Le, J. Egedal, and W. Daughton, "Two-stage bulk electron heating in the diffusion region of anti-parallel symmetric reconnection," Phys. Plasma 23, 102109 (2016).

${ }^{64}$ Y. V. Khotyaintsev, A. Vaivads, M. André, M. Fujimoto, A. Retinò, and C. J. Owen, "Observations of Slow Electron Holes at a Magnetic Reconnection Site," Phys. Rev. Lett. 105, 165002-+ (2010).

${ }^{65}$ S. Y. Li, Y. Omura, B. Lembège, X. H. Deng, H. Kojima, Y. Saito, and S. F. Zhang, "Geotail observation of counter directed ESWs associated with the separatrix of magnetic reconnection in the near-Earth magnetotail," J. Geophys. Res. 119, 202-210 (2014).

${ }^{66}$ M. Øieroset, D. Sundkvist, C. C. Chaston, T. D. Phan, F. S. Mozer, J. P. McFadden, V. Angelopoulos, L. Andersson, and J. P. Eastwood, "Observations of plasma waves in the colliding jet region of a magnetic flux rope flanked by two active $\mathrm{X}$ lines at the subsolar 
magnetopause," J. Geophys. Res. 119, 6256-6272 (2014).

${ }^{67}$ D. B. Graham, Y. V. Khotyaintsev, A. Vaivads, and M. André, "Electrostatic solitary waves with distinct speeds associated with asymmetric reconnection," Geophys. Res. Lett. 42, 215-224 (2015).

${ }^{68}$ C. Norgren, M. André, A. Vaivads, and Y. V. Khotyaintsev, "Slow electron phase space holes: Magnetotail observations," Geophys. Res. Lett. 42, 1654-1661 (2015).

${ }^{69}$ R. Wang, R. Nakamura, Q. Lu, W. Baumjohann, R. E. Ergun, J. L. Burch, M. Volwerk, A. Varsani, T. Nakamura, W. Gonzalez, B. Giles, D. Gershman, and S. Wang, "Electron-scale quadrants of the hall magnetic field observed by the magnetospheric multiscale spacecraft during asymmetric reconnection," Phys. Rev. Lett. 118, 175101 (2017). ${ }^{70}$ A. Zeiler, D. Biskamp, J. F. Drake, B. N. Rogers, M. A. Shay, and M. Scholer, "Threedimensional particle simulations of collisionless magnetic reconnection," J. Geophys. Res. 107, $1230-+(2002)$.

${ }^{71}$ H. Che, J. F. Drake, M. Swisdak, and M. L. Goldstein, "The adiabatic phase mixing and heating of electrons in Buneman turbulence," Phys. Plasma 20, 061205 (2013).

${ }^{72}$ G. Lapenta, S. Markidis, A. Divin, M. Goldman, and D. Newman, "Scales of guide field reconnection at the hydrogen mass ratio," Phys. Plasma 17, 082106 (2010).

${ }^{73}$ W. Daughton, V. Roytershteyn, H. Karimabadi, L. Yin, B. J. Albright, B. Bergen, and K. J. Bowers, "Role of electron physics in the development of turbulent magnetic reconnection in collisionless plasmas," Nature Physics 7, 539-542 (2011).

${ }^{74}$ D. E. Wendel, D. K. Olson, M. Hesse, N. Aunai, M. Kuznetsova, H. Karimabadi, W. Daughton, and M. L. Adrian, "The relation between reconnected flux, the parallel electric field, and the reconnection rate in a three-dimensional kinetic simulation of magnetic reconnection," Physics of Plasmas 20, 122105 (2013).

${ }^{75}$ W. Daughton, T. K. M. Nakamura, H. Karimabadi, V. Roytershteyn, and B. Loring, "Computing the reconnection rate in turbulent kinetic layers by using electron mixing to identify topology," Physics of Plasmas 21, 052307 (2014).

${ }^{76}$ R. B. Torbert, C. T. Russell, W. Magnes, R. E. Ergun, P.-A. Lindqvist, O. LeContel, H. Vaith, J. Macri, S. Myers, D. Rau, J. Needell, B. King, M. Granoff, M. Chutter, I. Dors, G. Olsson, Y. V. Khotyaintsev, A. Eriksson, C. A. Kletzing, S. Bounds, B. Anderson, W. Baumjohann, M. Steller, K. Bromund, G. Le, R. Nakamura, R. J. Strangeway, H. K. Leinweber, S. Tucker, J. Westfall, D. Fischer, F. Plaschke, J. Porter, and K. Lappalainen, 
"The FIELDS Instrument Suite on MMS: Scientific Objectives, Measurements, and Data Products," Space Sci. Rev. 199, 105-135 (2016).

${ }^{77}$ R. E. Ergun, K. A. Goodrich, F. D. Wilder, J. C. Holmes, J. E. Stawarz, S. Eriksson, A. P. Sturner, D. M. Malaspina, M. E. Usanova, R. B. Torbert, P.-A. Lindqvist, Y. Khotyaintsev, J. L. Burch, R. J. Strangeway, C. T. Russell, C. J. Pollock, B. L. Giles, M. Hesse, L. J. Chen, G. Lapenta, M. V. Goldman, D. L. Newman, S. J. Schwartz, J. P. Eastwood, T. D. Phan, F. S. Mozer, J. Drake, M. A. Shay, P. A. Cassak, R. Nakamura, and G. Marklund, "Magnetospheric Multiscale Satellites Observations of Parallel Electric Fields Associated with Magnetic Reconnection," Physical Review Letters 116, 235102 (2016).

${ }^{78}$ J. L. Burch, T. E. Moore, R. B. Torbert, and B. L. Giles, "Magnetospheric Multiscale Overview and Science Objectives," Space Sci. Rev. 199, 5-21 (2016).

${ }^{79}$ T. D. Phan, J. P. Eastwood, P. A. Cassak, M. Øieroset, J. T. Gosling, D. J. Gershman, F. S. Mozer, M. A. Shay, M. Fujimoto, W. Daughton, J. F. Drake, J. L. Burch, R. B. Torbert, R. E. Ergun, L. J. Chen, S. Wang, C. Pollock, J. C. Dorelli, B. Lavraud, B. L. Giles, T. E. Moore, Y. Saito, L. A. Avanov, W. Paterson, R. J. Strangeway, C. T. Russell, Y. Khotyaintsev, P. A. Lindqvist, M. Oka, and F. D. Wilder, "MMS observations of electron-scale filamentary currents in the reconnection exhaust and near the X line," Geophys. Res. Lett. 43, 6060-6069 (2016). 